\title{
ARTICLE
}

\section{Visual Workshop 2: a model viewer, editor and results display package for the ANSWERS shielding and criticality codes}

\author{
Adam J. Bird* and Tim C. Fry \\ AMEC Power and Process Europe, Kimmeridge House, Dorset Green Technology Park, Winfrith Newburgh, Dorchester, Dorset \\ DT2 8ZB, UK
}

\begin{abstract}
The goal of Visual Workshop is to be an 'Integrated Development Environment' for preparing, modifying, checking, running and analysing results from the shielding codes MCBEND and RANKERN, the criticality code MONK, and the reactor physics/criticality code WIMS. The functionality is being introduced in stages and this paper announces the release of version 2. The key features of Visual Workshop version 1 included: interactive $2 \mathrm{D}$ and $3 \mathrm{D}$ ray trace display of the model using the same routines as the analysis code, wire frame display of the model, built in editing, running and diagnostic capabilities and a simple results display. The main additional capabilities of version 2 are: Results display using 2D cell plots, 2D contour plots and 3D iso-surface plots overlaid on the model geometry; and display of event type and location and particle tracks overlaid on the model geometry.
\end{abstract}

\section{Keywords: results visualisation; graphics; MCBEND; RANKERN}

\section{Introduction}

The long term goal of Visual Workshop is to be an 'Integrated Development Environment' for preparing, modifying, checking, running and analysing results from the ANSWERS shielding codes MCBEND [1] and RANKERN [2], the criticality code MONK [3], and the reactor physics and criticality code WIMS [4]. The functionality is being introduced in stages and this paper announces the release of version 2 .

The main additional capabilities of version 2 are:

1. Results display using $2 \mathrm{D}$ cell plots, $2 \mathrm{D}$ contour plots and 3D iso-surface plots overlaid on the model geometry for the following result types:

- $\quad$ Scatter sampling probabilities (RANKERN).

- Importance map values and particle inflows (MCBEND/MONK)

- Flux and Response values (MCBEND/MONK)

- $\quad$ Action Tally values (MONK)

2. Display of event type, location and particle tracks overlaid on the model geometry:

- History Log and Collision log data (MCBEND/MONK)

*Corresponding author. Email: adam.bird@amec.com
- $\quad$ Source Log, Forced Flight log and Leakage Log data (MCBEND)

- $\quad$ Birth Store data (MONK)

\section{Description of Visual Workshop}

Visual Workshop is written in JAVA using SWING for the user interface components with the $2 \mathrm{D}$ and $3 \mathrm{D}$ ray trace capability provided by a 'Java Native Interface' link to FORTRAN code from MONK, MCBEND and RANKERN. The wire frame graphics display is provided by the Visualisation Toolkit (VTK) [5].

Visual Workshop consists of four tabbed components; 3D Wire view, 3D Ray view, 2D Ray view and Launchpad view with an overview component on the left. Optionally a sub-view component can appear below the four main components. Figure 1 shows Visual Workshop with the sub view containing the input editor and the wire frame tab selected.

The Launchpad view handles selection of the MCBEND version and selection of data library versions - this is largely automatic and based on the requirements of the input data and running calculations. A run monitor is provided to 'watch' a running calculation and catch any messages that would appear on a console. A queuing tool is available allowing submission of a series of calculations in batch mode.

The 3D Wire view displays the geometric bodies used to construct the model as described in the Fractal Geometry (FG) package of MCBEND. The display 
supports solid, translucent and wire frame modes, cut-away planes and selection of components to display. Additional code version features can be viewed, for example a MCBEND Unified Tally mesh.

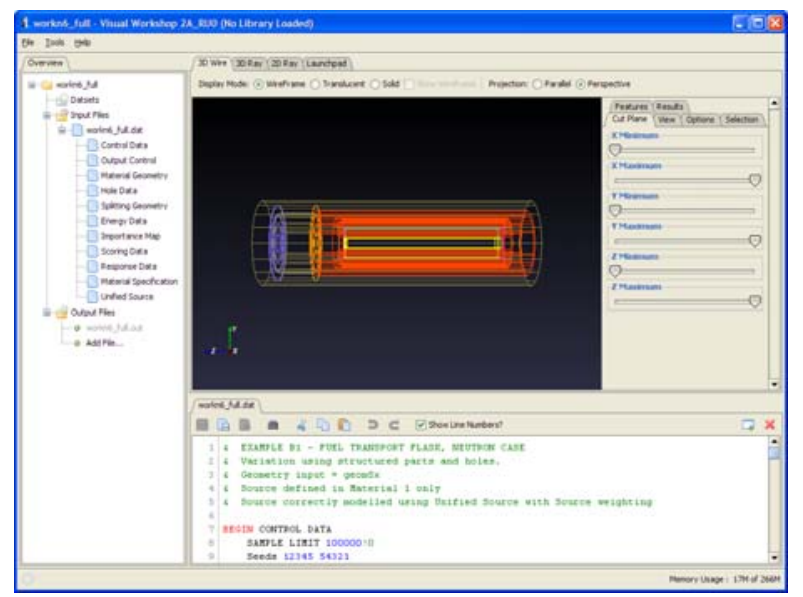

Figure 1. Visual Workshop with the sub view containing the input editor and the wire frame tab selected.

The 3D Ray view displays the model using the FORTRAN tracking routines that MCBEND uses to run a calculation. This means it is the most accurate representation of what the model really is. Also 'Hole' ${ }^{\text {a }}$ geometries are accurately displayed. The 3D ray trace display supports cut away planes and selective exclusion or transparent rendering of components. Errors in the model can be automatically detected and displayed. Many code version features can be viewed as overlays on the display. The display is initially rendered at a low resolution then progressively refined, allowing the 3D ray trace display to be fully interactive and rendered in real time.

The 2D Ray view displays a slice through the model. In other respects it is similar to the 3D ray capability.

The overview component lists the files associated with a calculation and provides shortcut links to key sections of the input data.

The Input Editor provides a full-featured text editor including syntax colouring. Changes that are saved using this editor will automatically result in updates to the displays.

The sub-view component can contain the input editor, output file viewers or graphed results, among other things. These may optionally be detached as separate windows.

\section{Code developments}

A lot of development work has gone into the production of Visual Workshop 2 not only to add important new features, but also to improve the features

\footnotetext{
${ }^{a}$ Hole geometries in MCBEND use Woodcock tracking which enables potentially complex shapes to be efficiently modelled, because they are not defined by simple bodies they can not be displayed by the wire frame.
}

in version 1. First, improvements to existing features are covered:

The user interface has been improved to make the controls for the three display types; 3D Wire Frame, 3D Ray trace and 2D ray trace; more consistent. Feedback from version 1 has helped guide changes made for version 2 .

The parser, which is used for reading and interpreting the input data for MONK, MCBEND and RANKERN, has been refined and developed to understand some of the more complex syntax available in the ANSWERS assessment codes, for example:

- Looping parameters.

- Multiple File Input.

- $\quad$ Processing from a 'datsets' file.

Visual Workshop uses a system of self testing, version 2 has seen improvements and extension to this set of 'auto-tests'. These are described in a section 5 .

In anticipation of the next release of the ANSWERS codes MONK and MCBEND Version 2 of Visual Workshop understands and can display features which will be new with these codes.

The major new developments are as follows, and are described in more detail below:

- $\quad$ Use of the Visualisation Toolkit (VTK) to provide the 3D wire frame display.

- Results display in the form of cell plots and surface contours.

- Event location and particle history display.

\subsection{VTK graphics}

Version 2 of Visual Workshop uses the VTK toolkit to provide the wire frame display. This has improved the quality of the graphical display and enabled development of the results display features. However, these improvements are not for free, version 2 has larger memory requirements and works better with more powerful graphics hardware than was required for version 1.

\subsection{Results visualisation}

This is a major new feature in version 2. Visual Workshop is able to scan an output file and extract results data which can then be displayed overlaid on the model in the wire frame display.

Additional display styles have been provided; 'Neutral' displays the model in grey shades leaving the results in colour to enable easier visualization and 'Report' which is 'Neutral' with a white background for use in documents.

The following result types are supported in version 2 .

- $\quad$ Scatter sampling probabilities (RANKERN).

- Importance map values and particle inflows (MCBEND /MONK)

- $\quad$ Flux and Response/Dose values in Unified Tally (MCBEND /MONK) 
- $\quad$ Action Tally values in Unified Tally (MONK)

These results can be plotted as a 3D contour or iso-surface (Figure 2), a 2D contour plane plot (Figure 3 ) or a $2 \mathrm{D}$ cell plot. The cell plot displays the results by cell as they are tallied in MCBEND whereas the contour plots interpolate the contour based on the mid point of a cell.

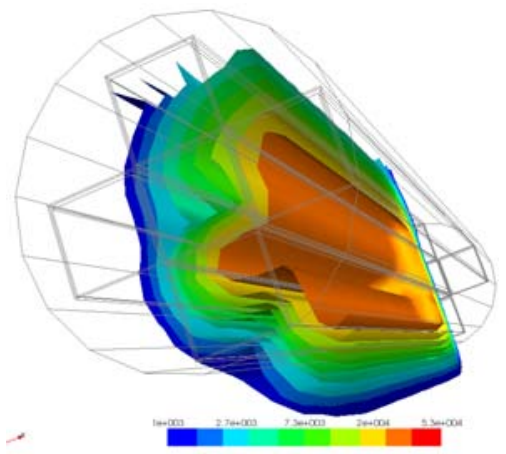

Figure 2. 3D Neutron dose contours inside a transport flask.

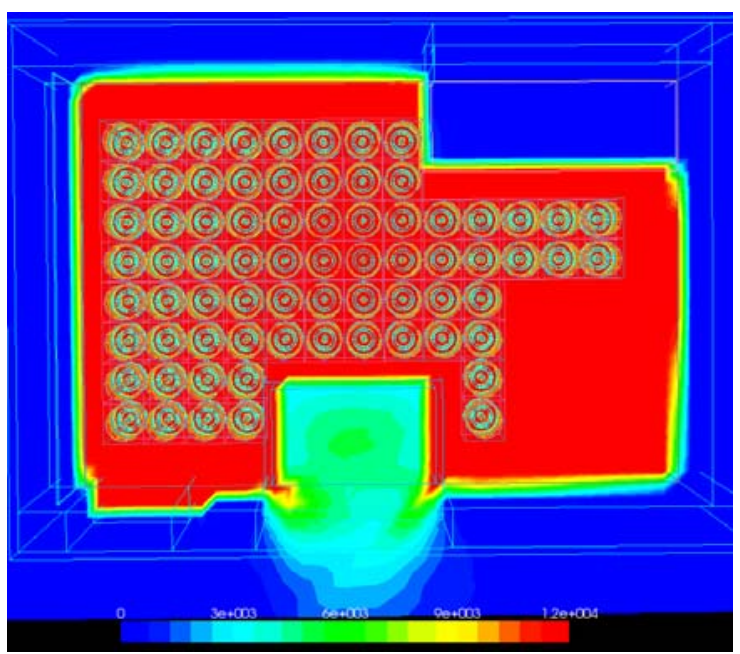

Figure 3. 2D Gamma-Ray dose contours of a dry storage facility.

\subsection{Event location and track visualisation}

The ANSWERS codes MCBEND and MONK are able to write event locations and particle history information to a range of files. Visual Workshop version 2 is able to read these files and display the particle histories on the $3 \mathrm{D}$ wire frame, 3D ray trace and $2 \mathrm{D}$ ray trace images.

The event files supported by version 2 are:

- $\quad$ History Log and Collision log data (MCBEND/MONK)

- $\quad$ Source Log, Forced Flight log and Leakage Log data (MCBEND)

- $\quad$ Birth Store data (MONK)

The events and tracks can be selectively displayed by sample number, energy, weight, time, location, reaction type and nuclide or material the reaction occurs with. Figures 4 and $\mathbf{5}$ show examples of particle track display.

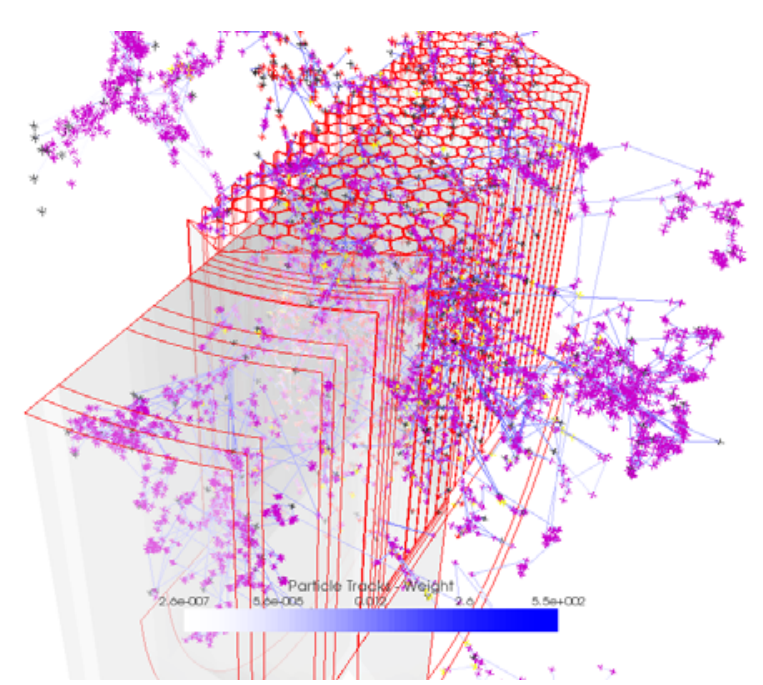

Figure 4. History log coloured by event weight on translucent wire frame of reactor with geometry cut away.

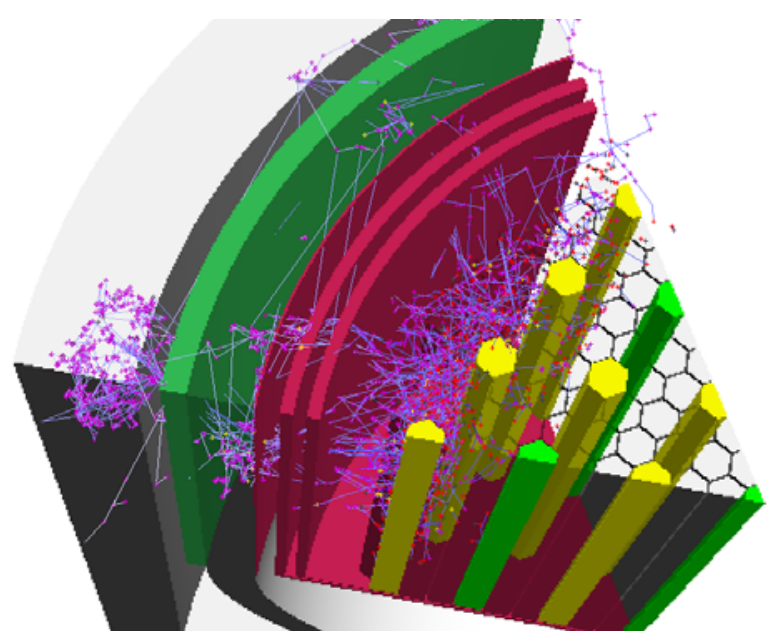

Figure 5. History log coloured by event weight on 3D ray trace with cut away geometry, transparent materials and hidden line removal.

\subsection{Support for development features}

Visual Workshop provides ray traced images of the model using the particle tracking FORTRAN used by the ANSWERS codes MCBEND, MONK and RANKERN. The correct FORTRAN dynamically loaded library is chosen at runtime based on the code version selections in the Launchpad tab. The de-coupling of the GUI and the ray trace functionality allow Visual Workshop version 2A to support and test some development features.

The development features include:-

Import of CAD geometry in IGES format [6]: This provides support for complex surfaces with no approximation. (Figure 6)

Import of polygon surface geometries: This provides high performance support for complex surfaces with some geometric approximation. (Figure 7) 

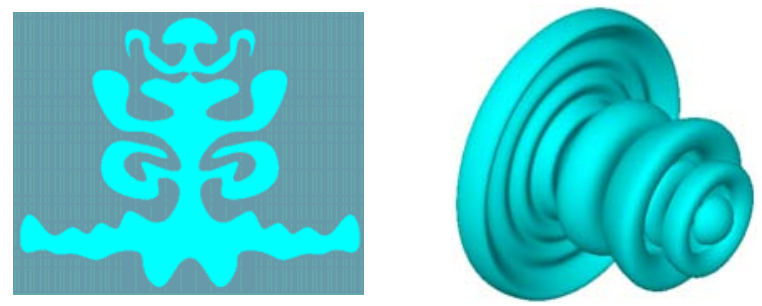

Figure 6. Example of an imported IGES geometry.

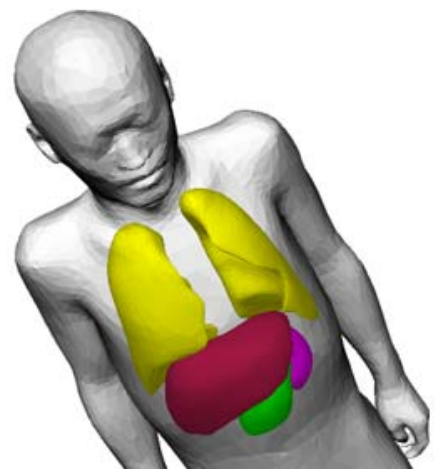

Figure 7. Example of a polygon surface geometry.

\section{3D Wire frame stereo viewing}

Visual Workshop version 2A includes functionality to allow the contents of the 3D Wireframe View to be displayed in stereo using a compatible combination of graphics card, monitor and stereo glasses.

The functionality uses OpenGL Quad Buffered Stereo to display stereo images. While a number of manufacturers produce hardware suitable for viewing this type of stereo display, ANSWERS uses and suggests customers use hardware compatible with the NVIDIA 3D Vision Pro system.

This is currently development status feature which can be enabled using a command line argument if users with suitable hardware wish to try the functionality.

\section{Testing}

Most of the functionality is tested using built in self testing routines. This means that the user testing can be kept minimal and is associated with 'does the button work?' rather than 'does the process invoked by the button work?'. Many of the tests involve comparing generated images which is done in an operating system and graphics capability independent way. For example the event log display tests involve 220 image comparisons.

All of the test inputs and reference files are shipped as part of the standard package so Visual Workshop can be instructed to start in test mode and produce an HTML test report.

\section{Conclusion}

Visual Workshop has undergone significant developments since the release of version 1 . It has made large steps towards the long term goal to be an 'Integrated Development Environment' for preparing, modifying, checking, running and analysing results from the ANSWERS codes MCBEND, MONK and RANKERN. Feedback from customers has been very positive saying have found the results and event log display features particularly useful.

\section{Acknowledgements}

This acknowledges the input of the ANSWERS team who have all contributed to the development of Visual Workshop.

\section{References}

[1] P. Cowan, G. Dobson and J. Martin, Release of MCBEND 11, Proc. $12^{\text {th }}$ International Conference on Radiation Shielding (ICRS-12) and $17^{\text {th }}$ Topical Meeting on Radiation Protection and Shielding (RPSD-2012), Nara, Japan, (September 2012).

[2] S. Chucas and I. Curl, Streaming Calculations using the Point-Kernel Code RANKERN. Proc. $9^{\text {th }}$ International Conference on Radiation Shielding (ICRS-9), Tsukuba, Japan, (October 1999)

[3] M. Armishaw, N. Davies and A.J. Bird, The ANSWERS Code MONK - A new approach to scoring, tracking, modelling and visualisation. Proc 9th International Conference on Nuclear Criticality Safety (ICNC 2011), Edinburgh, Scotland, (September 2011)

[4] T. Newton, G. Hosking, L. Hutton, D. Powney, B. Turland and E. Shuttleworth, Developments within WIMS10, Proc International Conference on Physics of Reactors "Nuclear Power: A Sustainable Resource" Casino-Kursaal Conference Center, Interlaken, Switzerland (September 14-19, 2008)

[5] Visualisation Toolkit, An open-source software system for 3D computer graphics (See http://www.vtk.org/.)

[6] A. Bird and A. Kyrieleis, Experience using models imported from CAD software for shielding calculations in MCBEND, Proc. $12^{\text {th }}$ International Conference on Radiation Shielding (ICRS-12) and $17^{\text {th }}$ Topical Meeting on Radiation Protection and Shielding (RPSD-2012), Nara, Japan, (September 2012). 